\title{
Multimodal construction of soccer-related humor on Twitter and Instagram
}

\author{
Thomas Messerli and Di Yu
}

\section{Introduction}

Technology-mediated communication, particularly within popular social media sites, grants users active roles and participatory access to its content (Chovanec and Dynel, 2015). In this chapter, we analyze specialized soccer humor channels on Twitter and Instagram and explore the multimodal construction of soccer-related humor on these sites, and how that humor is taken up in followers' comments. Accordingly, our research questions are: (1) How is humor constructed by original posters (OPs) on soccer-related Twitter and Instagram channels? (2) How do other users/commenters react to humorous tweets and Instagram posts, and what forms of humor support can be observed in this context?

In the analyzed posts, the broadcaster, i.e., the "'followable' party that makes talk available to recipients" (Draucker, 2015, p. 49), uses text, images, videos, audios, and GIFs to construct humorous incongruities and invite their followers to laugh with them about a specific target-usually an individual player or a soccer club. In doing so, broadcasters establish involvement in the sense of Tannen (2007), i.e., they use the specific linguistic and non-linguistic materials at their disposal to create and maintain an emotional connection between their followers and themselves. In our analysis of OPs, we will thus focus on the ways in which visual and textual components are combined and reused by posters to construct soccerrelated humor. As the analysis will show, the soccer frame is activated more directly by referring to or combining constituent elements (including participants and events) of the beautiful game, or more indirectly by repurposing aspects from other domains and forcing a shift towards the soccer frame. Encoded in the posts is an assumption by OPs that their common ground with the recipients extends to even minor events in recent soccer games of the major European leagues as well as of European international competitions like the Champions League. While the multimodal construction of humor in itself may well be shared with humor in other domains, the thus constructed soccer-related incongruities are special because they 
can appeal to a wider audience despite the extensive knowledge they require to be successfully processed as humor.

In a second step, we will then investigate the comments that followers post in response to the OP and establish how reactions more generally and humor support more specifically are encoded in these comments. By demonstrating how original posters construct humor and how other users respond to and support humor, we provide further evidence of the complexity of humor construction and collaborative nature of communication on social media. These comments also support the successful uptake of humor and thus the understanding and appreciation of the soccer-related details that the OPs made use of.

Before presenting the findings of these empirical analyses in the fourth and fifth sections ("Analysis 1: Humor construction in original posts" and "Analysis 2: User responses to humorous soccer-related posts"), we will briefly establish the theoretical framework that informs our understanding of computer-mediated communication (CMC), interaction and of humor therein (second section: "Background"), as well as the data and methodology on which these findings are based (third section: "Data and method").

\section{Background}

\section{Communication on social media}

Previous research has noted some prominent properties of the interactions occurring on social media in various aspects such as the role of the original poster (OP), the participation framework, the role of media artifacts, and the possibility to engender civil participation. Draucker (2015) for example, in analyzing the structure of interaction and participation on Twitter, identified the role of the 'broadcaster' as one that complements the production roles in the Goffmanian tradition and better captures the complexity of interactions occurring on the medium of Twitter. It is suggested that the broadcaster can not only be distinguished as the animator, the author, or the principle, but can also serve as an accountable party that makes talk available. It has also been noted that, despite participants' spatial separation, interaction on social media enables an "infinite number of potential participants" (Chovanec and Dynel, 2015) to become an automatically ratified audience.' As Chovanec and Dynel (2015) point out, instead of being simply a passive recipient, any audience member also has the potential of taking on other participation roles and becoming active producers themselves, which leads to a shift from simply dyadic interaction to dynamic co-creation. Besides the complex roles of participation, social media sites also provide the use of media artifacts, which has also become a noteworthy phenomenon. Typical artifacts, such as memes, pictures, videos, GIFs, Vine video loops, are said to help express one's perspectives 
and facilitate the dialogue between parties with different opinions, which essentially contributes to creating "a plurality of coexistence and interaction" (Milner, 2013, p. 2358). Overall, social media as an interactive and democratic platform has been found to be consequential for engaging the public and creating opportunities for civil participation (Butterworth, 2014). More specifically regarding soccer, it has been found that media discourse contributes to normalizing fandom and fanaticism and spectacularizing and commercializing soccer events (Lee, 2005). Cleland (2014) also found that soccer-related social media can become platforms for antiIslamic and anti-multiculturalism racist discourse. As will be seen, our data contains some aspects that may be regarded as conservative or even homophobic, but generally is of an entirely more playful nature, bringing together posters and commentators that jointly engage in topical humor about the sport they are interested in.

\section{Theoretical approach to humor}

We follow what can be described as an essentialist view of humor in this chapter, which is interested in the mechanisms and affordances the broadcasting party employs in the creation of humor in the original posts, as well as in how followers encode their reactions to humorous posts in their comments. Within the classical three strands of humor-relief, superiority, and incongruity (see e.g., Attardo, 1994) - this study adheres to a definition of humor based on incongruity and resolution, which is informed by Suls's (1972) model of humor processing. Humor is understood first and foremost as a cognitive phenomenon that occurs when a surprising stimulus runs against recipient expectations: the stimuli that recipients encounter evoke a particular narrative frame, i.e., knowledge structures that lead to vague expectations as to what events are likely to follow. Subsequent stimuli, e.g., in the form of narrative events, are measured against those expectations, and humor ensues if a stimulus is incongruous with recipient expectations, and if that incongruity can be resolved by finding a rule that explains its presence. While humor processing ultimately takes place in the minds of the recipients-in this case the followers that read and react to the posts-the focus of this study is on the basis of these processes in the stimuli that are encoded in posts on social networks.

\section{Humor in interaction and CMC}

The forms of humor in interaction are found to include but are not limited to joke telling, anecdotes, wordplay, and irony (Norrick, 2003). Typical features of conversational humor include overlapping speech, coconstructions of utterances, metaphorical language, teasing, sarcasm, linguistic put-downs, higher pitch or volume, increased speech rate, and 
significant pauses (Attardo et al., 2011; Coates, 2007). In conversations, it has been found that humor can be accompanied and signaled by paralinguistic cues such as the speaker's or the listener's laughter or smile, change in tone of voice, change in pitch or rhythm (Glenn, 2003; Norrick, 2003; Jefferson, 1979). For conversations within CMC, it has been suggested that humor is used to establish group solidarity and identity as users navigate social life on online platforms (Baym, 1995). Evidently, not all of the mentioned features will be found in humor instances on social media. As our discussion of humor comments will show, however, the posters and commenters use the particular linguistic and paralinguistic cues afforded to them by the respective social media platform in order to encode humor and humor support.

\section{Humor support strategies and responses to humor}

Also relevant to this project, particularly the second research question, which addresses the uptake of humorous posts in subsequent comments, is previous work on humor support strategies and responses to various types of humor. Treating humor as an interactive phenomenon, Hay (2001) notes commonly observed humor support practices including laughter and echoing or contributing more humor. Overlap and other involvement strategies can be used to show appreciation and enthusiasm. In the case of selfdeprecating humor, often sympathy or contradiction are found as responses. In experimental settings, Bell (2013; 2009) also observes responses to failed or incomprehensible humor, which is particularly interesting for the purpose of this project. She discusses a few typical responses to failed humor including signaling recognition while expressing the lack of appreciation, laughter and fake laughter, metalinguistic comments, interjections, evaluative responses, rhetorical questions, and sarcasm. In the case of incomprehensible humor, she also discovered that typically recipients respond with expressions of non-understanding, smile, laughter, silence, repetition of the punch line, assessment of jokes, and request for explanation.

\section{Soccer as a semantic frame}

In order to address the specificity of the humorous incongruities we find in our data for soccer, we will describe it as a semantic frame by using the terminology and existing framework that is implemented in FrameNet (see e.g., Fillmore and Baker, 2009). Understood from this point of view, soccer can be described as a form of competition, which as core elements involves a set of participants, the players, as well as many less central elements that include the venue (the pitch that it is played on), the additional participants that are involved (e.g., referees), and a particular purpose (winning 
this particular game, winning a competition, etc.). For the particular type of soccer that our tweets and Instagram posts relate to, we can further specify that we talk about the kind of top-tier men's professional soccer that is broadcast on television and seen by a global audience, either because it belongs to a soccer competition that is itself international, or because it is part of a national competition whose appeal goes beyond national borders (e.g., the English Premier League).

When we talk about soccer-related humor in our discussion, we thus mean humor that uses the core and non-core elements of internationally broadcast professional soccer as part of its setting. In particular, we find that both the verbal and visual elements of the individual humorous posts activate particular aspects of the soccer frame and work in relation to expectations based on knowledge of soccer, the domain of professional soccer and the conventions of televisually broadcast soccer. As will be shown, all of these knowledge structures are assumed by original posters to be part of the communal common ground of their recipients, which in turn renders them affordances for humor construction. At the same time, these processes thus also construct a particular audience-we find no traces of explication of any post elements to recipients who may not be familiar with them.

Based on this study alone, it is not possible to decide to what extent the patterns we find here are shared with other semantic frames. However, it is striking that the topicality of the channels we investigate here translates into exclusively topical humor that is either inherently situated entirely within the soccer frame or transferred there with the help of processes that we will discuss in the fourth section ("Analysis 1: Humor construction in original posts"). It can be added that while the mechanisms of humor construction we find here are linked more generally to the affordances offered by Twitter and Instagram, their concrete realization builds on a number of aspects that in their combination are particular to the soccer frame. These aspects include the international popularity of the game that leads to similar televisual representations across the globe; the fact that it is a playful competition that is received as a form of entertainment and therefore requires little work to be made the topic of humor; and the appearance of particular participants (e.g., the player Lionel Messi) as well as more generic roles (e.g., referees), whose typical traits and behavior are known to an international audience.

\section{Data and method}

In order to answer our research questions, we collected data from four public soccer-related social media accounts, two each on Twitter and on Instagram, in the first week of June 2016. Our analyses focus on the original posts from each account, as well as on comments by other users in 
response to each post, which lets us address both humor construction by original posters (OPs) and humor uptake, including humor support, in the subsequent response to these original posts. Given our interest in the multimodal construction of soccer-related humor and its uptake in the social media, we started by searching for topical Twitter and Instagram accounts and based on their popularity selected FootyHumour and Footy_Jokes 02 on Twitter, and footballjokes and perksoffootball on Instagram. This can be regarded as a form of cluster sampling: We assume that posters on these social platforms do in most cases not create the individual elements that constitute their posts and will instead (re-)assemble pre-existing pictures, GIFs, videos, and even texts in order to create posts they then share with their followers. Thus, the selection of humor we find on each account should be a heterogeneous subset of humorous soccer-related posts that are being shared on each of the two social networks. Indeed, we found that while there were differences between Instagram and Twitter, the two accounts on each platform were very much comparable with regard to the type of posts they contained.

Since this is an exploratory study interested in creating a typology of multimodal humor construction on social networks, we were content with a relatively small data set in our first analysis and accordingly limited the number of posts to a sample of 20 from each account. For the resulting 80 posts, we saved the URL where each of them was accessible, the texts each of the posts contained, and a screenshot of the post as it presented itself in June 2016. We mention this here because a first observation we can make is that these posts have various degrees of fixity. Whereas the majority of posts by three of the four accounts could still be accessed in January 2017, Footy_Jokes 02 has since been closed. Similarly, some of the comments we collected for the second analysis are still publicly available, while others have been removed-mostly because the accounts of the commenters have since been deleted.

Having selected the posts, we analyzed them qualitatively for the ways in which they multimodally create humorous incongruities, which resulted in a non-exhaustive list of humor construction strategies that will be presented and discussed based on illustrative examples in the section "Analysis 1: Humor construction in original posts." Based on the finding of the first analysis that each of the two Instagram and Twitter accounts were comparable clusters of humorous soccer-related posts on the respective platform, we again decided on cluster sampling for the second analysis and limited our study to the 643 comments made in response to the posts of the Twitter account FootyHumour and the 1292 comments to perksoffootball on Instagram. These comments were analyzed qualitatively and bottom-up, i.e., without preconceived notions about what type of comments we as researchers expected. The results of the second analysis are presented in the section "Analysis 2: User responses to humorous soccer-related posts." 
We want to conclude this section with a brief note on research ethics. First, we agree with Bolander and Locher (2014, pp. 16-17) that ethical issues need to be taken into account by researchers that work with data, which includes data that are publicly available on the internet. The data we analyze here are public not only in terms of their accessibility, but also in terms of their content, and we have found no evidence in posts or comments that would indicate in any way that the poster or commenter would want their message to be understood as private. Moreover, the analyzed data can be described as light-hearted interaction on a public and nonsensitive topic, and it is not conceivable how our research could be disadvantageous in any way to any of the posters and commenters whose contributions appear in our data. We therefore decided that even in the absence of consent, analyzing the data and presenting them in examples as we do here is well within what we deem to be ethically sound research practice. The public nature of the data notwithstanding, all personal information that is not immediately relevant to our analyses has been greyed out in the figures.

\section{Analysis I: humor construction in original posts ${ }^{2}$}

As outlined in the previous section, the initial analysis of soccer-related humor on Twitter and Instagram focused on the original posts by FootyHumour and Footy_Jokes02 on Twitter, and footballjokes and perksoffootball on Instagram. Since the analyzed posts are multimodal in the sense that they make use of one or several pictures and of short texts, which are present in the form of captions or post texts, we will present the types of incongruities that occur in the data categorized by the primary locus of the incongruity. This can be either in the pictorial content, in the textual content, or in equal parts in each of the two.

\section{Picture-related incongruities}

As we have mentioned, the primary mode of Instagram is visual, which is to say that every post will by default contain at least one picture or video. Twitter posts, on the other hand, may consist of text only. However, due to the role that television broadcasts play in the dissemination of the type of soccer we focus on here, we expected only marginally less multimodality on Twitter than on Instagram. This was confirmed by the analysis of our data, with 39 of 40 tweets including a picture or video. The only exception is a tweet that uses only text to emulate a scoreboard and thus nonetheless creates a visual effect. Not only do pictures occur in the data, they are often also the primary locus of the humorous incongruity, and the following five OP-strategies are ways in which pictures are exploited for soccerrelated humor. 
Strategy 1: incongruities in pictures

When it comes to the work that is involved for the poster, the simplest strategy for constructing a picture-based incongruity is to find pre-existing humor and share it with followers. One or several pictures or videos that contain incongruities are presented either without text, or with a caption or post that highlights the incongruity the target audience is meant to look for in the post.

Figure 11.1 illustrates this by presenting what it labels the "Best kisses in football history": It includes three pictures of soccer players kissing other players or male or female fans, with the poster asking their followers to choose their favorite of the three. The pictorial incongruities in Figure 11.1 can be interpreted on different levels. For starters, the combination of the frames of soccer and romance is untypical and thus surprising. At its core, soccer as a competition is about those actions that allow the group of participants that play for one team to win against the other team using the means that the rule of the game affords them. Including more peripheral activities, celebratory scenes when significant steps towards that goal have been taken are also expected, in which case elements of the frames of celebration as a type of social event and friendship as a type of personal relationship are also evoked. This does not, however, include unambiguously romantic actions. Given the strong heterosexual bias shared by the majority of soccer players (and athletes more generally) and many of their fans, the two pictures displaying kisses between two men may be perceived

\section{Best kisses in football history}

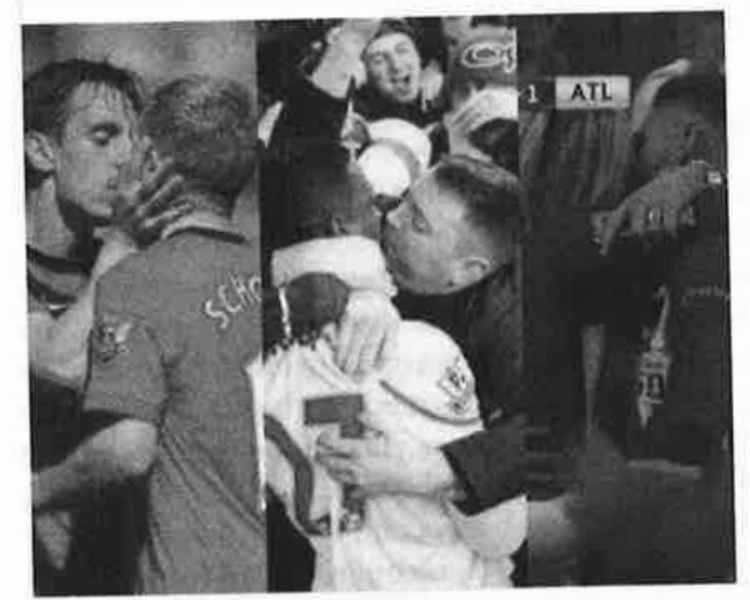

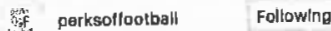

perkeolfootball Whlch one is your fevourite?

Figure 11.1 Incongruities in pictures. Perksoffootball, I June 2016. Instagram. 
as particularly incongruous with expectations. ${ }^{3}$ While comparatively little effort is required for the poster in these cases-they merely find rather than create incongruities-the inclusion of the third image (a player kissing a woman) and the explicit invitation by the OP for followers to choose between the three images ("Which one is your favourite?") indicate that the inherently incongruous material is carefully positioned in a way that highlights its incongruousness. It has to be added that this strategy is constrained by the availability of pictures and videos that can be found and distributed: In order to be relevant for these accounts that are dedicated to soccer-related humor, postable pictorial content needs to be both humorous and belong to the domain of soccer.

\section{Strategy 2: captions recontextualizing incongruities in pictures}

The second strategy apparent in the data is related to the first one insofar as here, too, the posted picture already contains a humorous incongruity and thus can be said to be humorous in and of itself. However, in this case, the incongruity does not or not entirely reside within the domain of soccer, which is why work has to be done by the poster to make it relevant. In Figure 11.2, for instance, the pre-existing incongruity in the picture contrasts the frame of soccer with that of the snowman, an inanimate object that bears some iconic resemblance to a human agent. This contrast is only activated, however, when the poster establishes a metaphorical connection to Arlético Madrid's Slovenian goalkeeper Jan Oblak. In the decisive penalty shoot-out of the UEFA Champions League final, which had taken place three days before this Instagram post was made, Oblak failed to stop

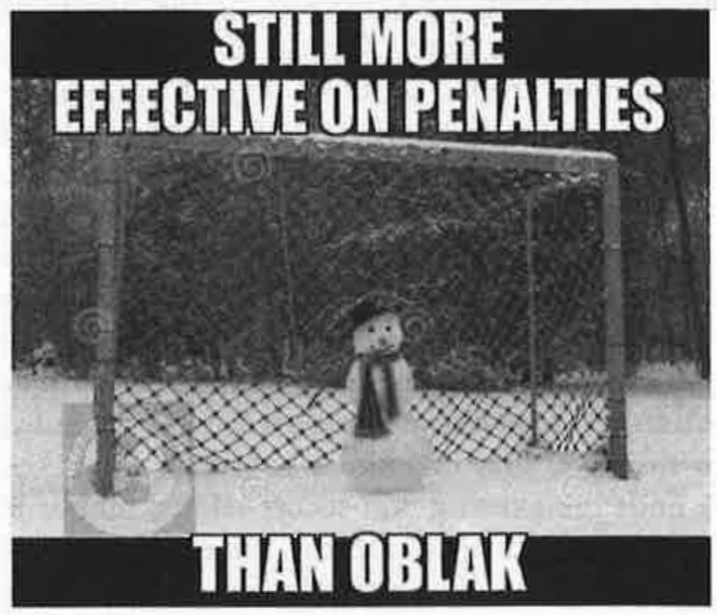

Following

perksoffootball \#oblak

Figure 1/.2 Recontextualizing incongruity. Perksoffootball, 31 May 2016. Instagram. 
any of his opponents' penalties. The post is thus an attempt to make fun of Oblak, who is made responsible for the resulting defeat against city rivals Real Madrid. The picture and the reference to effectivity on penalties in this case only require generic knowledge of soccer. Recipients need to know that the participant that stands between the goalposts is typically the goalkeeper and that he or she attempts to anticipate the direction of the ball and stop it before it crosses the line. They realize that for a snowman to be called more effective than a goalkeeper, that goalkeeper must do worse than standing still. This instance of hostile humor is then merely linked to the name 'Oblak', which evokes not only the particular goalkeeper, but also his performance in the recent final.

Figure 11.3 from the Twitter account FootyHumour follows the same strategy, but uses the caption to liken a video of a dancing boy to the joy Manchester United fans must have experienced when they learned about star player Zlatan Ibrahimović joining their club, which was announced around ten days before this post. Interestingly, the OP accidentally or consciously left a previous caption, "Chelsea fans right now...," in place, which shows that the same video on Vine had previously been used to establish a link to another club, Chelsea, and metaphorically render their reaction to another event.

Strategy 3: reinforcing incongruities in pictures by juxtaposing them with other pictures

Another similar strategy employed by OPs in our data is to find a humorous soccer-related picture or video and then to combine it with new pictorial content in order to reinforce the humorous effect. This strategy can be described as a combination of the first two, since it on the one hand makes use of a pre-existing incongruity from the domain of soccer, and on the other hand uses additional content from a different domain for humorous effect. Figures 11.4 and 11.5 are two stills from a video that was posted by perksoffootball and used to create humor in this fashion: The video consists of two parts, each of them around two seconds long. The first part, illustrated by Figure 11.4, is a moment taken from the live broadcast of a recent soccer game, in which the referee is repeatedly sticking his tongue out. The second part (Figure 11.5) simply adds video of a goat doing similar movements with its tongue. As was done in the Snowman/Oblak example (Figure 11.2), the post establishes a connection between soccer and a different semantic frame, in this case that of animal behavior. However, whereas the former example finds pre-existing non-soccer related humor in order to make fun of a soccer event, in this case the pre-existing visual incongruity is already topical and could be posted on its own, and the juxtaposition with another domain merely serves to emphasize the unexpectedness, in this case of the referee's behavior. While our analyses here 
Man Utd fans after hearing that Zlatan is joining them!

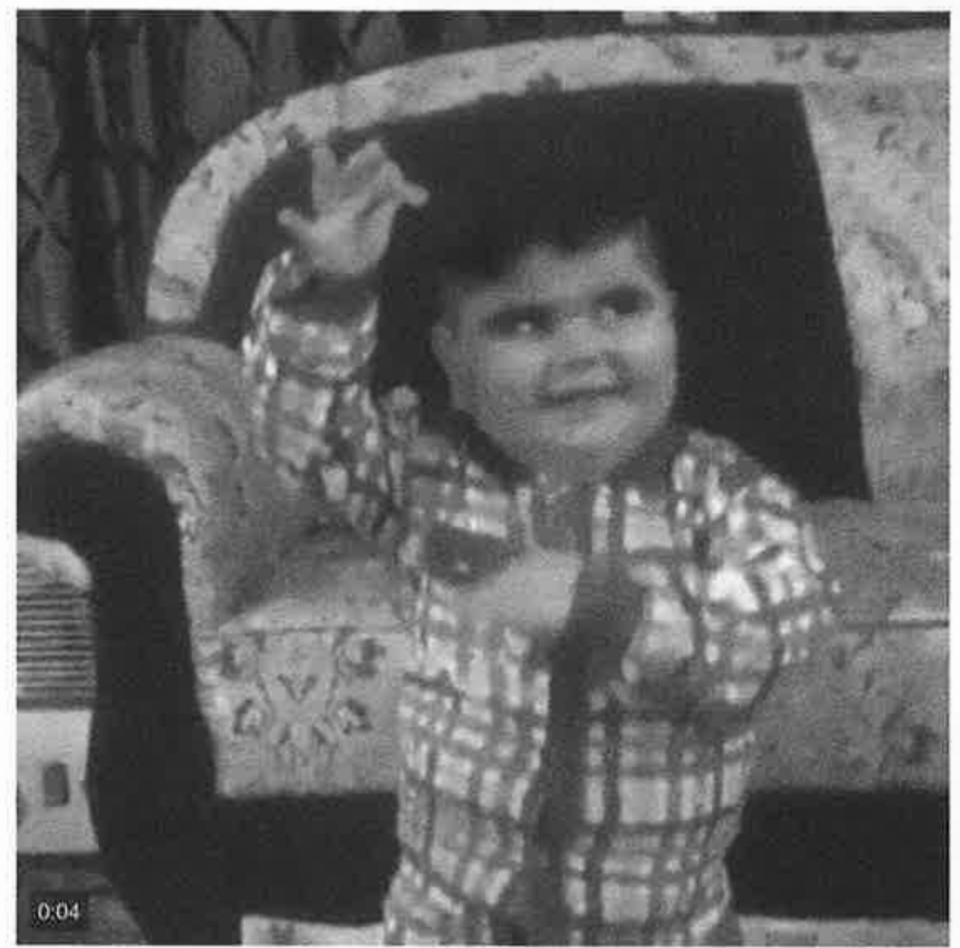

Follow @FootyHumour on Twitter's post on Vine Chelsea fans right now...

View on Vine

Figure 1/.3 Reinforcing incongruities in pictures. Footyhumour, 3 June 2016. Twitter.

leave aside aspects of typicality, we would assume this third strategy to be rarer than the first two because it has the same constraints as the first one, i.e., it is limited to pre-existing humorous and soccer-related material, and at the same time requires extra work by the poster like the second strategy.

\section{Strategy 4: incongruous collages}

The strategies that were discussed so far have in common that they do not alter the individual images they use to construct humor. This is different in 


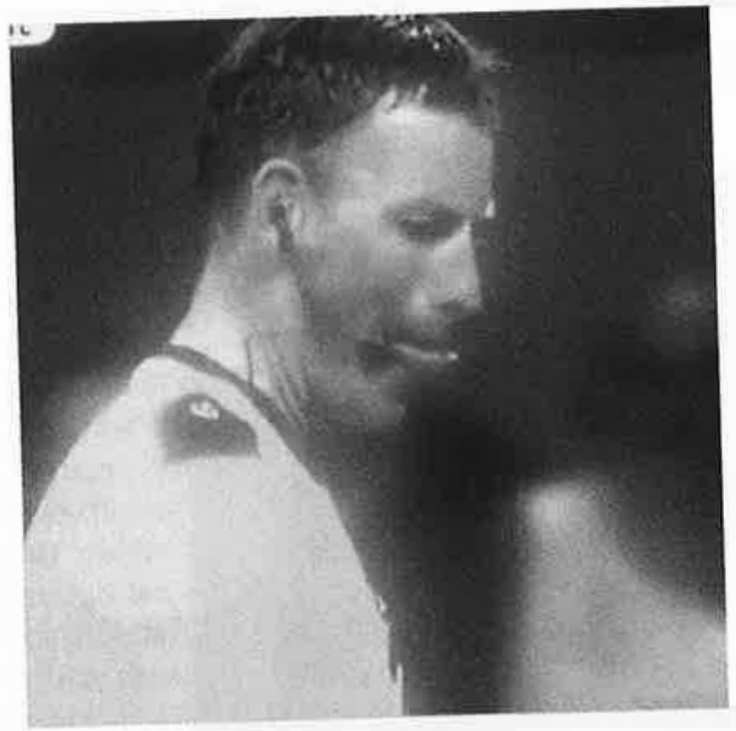

Following

perksoffiootball Helattenburg

Figure 11.4 Incongruous action. Perksoffootball, 31 May 2016. Instagram.

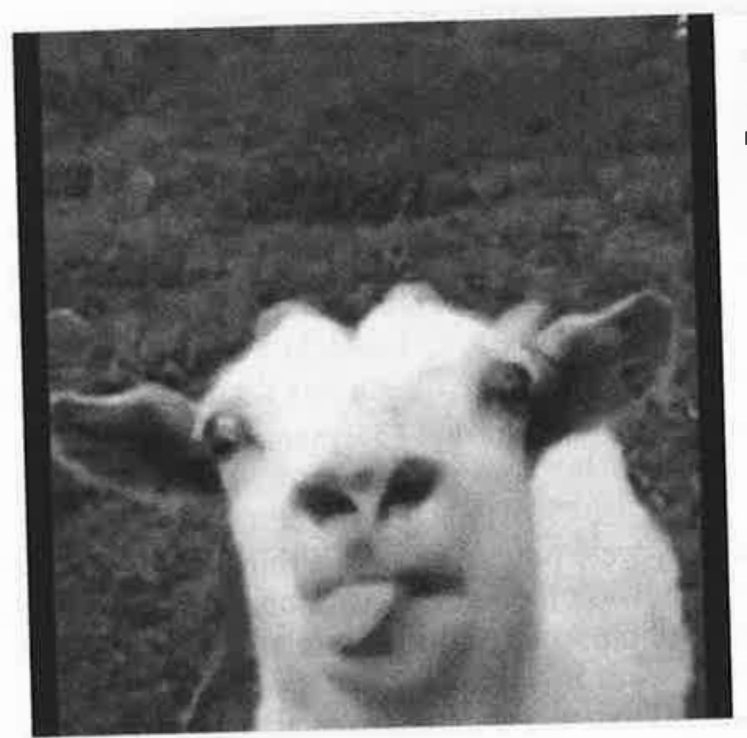

\section{Forksoffootball Following \\ perksoffootball Aclettenburg}

Figure 11.5 Juxtaposing incongruities. Perksoffootball, 31 May 2016. Instagram. 
the strategy that we have here termed incongruous collages. Conceptually, it is again a case of establishing a metaphorical link between different events. However, rather than achieving this effect in a linear fashion, by juxtaposing different elements, the images themselves are manipulated in order to map the two domains. Figure $\mathbf{1 1 . 6}$ presents an example from footballjokes, in which the underlying picture shows an Atlético Madrid player running to the sidelines to embrace a female supporter, while a man in a white shirt holds on to the player's arm. This image has been altered by the poster in a very simple fashion, by inserting a logo of the soccer club Real Madrid to cover the Atlético player's head, a picture of the UEFA Champions League cup to cover the female fan's face, and an Atlético logo to cover the face of the man in the white shirt. Without the manipulation, there is of course nothing incongruous about the celebratory scene. However, for those aware of the context of the UEFA Champions League final between Real and Atlético that was already mentioned, a relation of opposites between the two levels of the picture emerges. In the original picture, an Atlético player is about to embrace an Atlético fan, while another fan acts as a bystander desperate to quite literally grab the player's attention; in the collage, the Atlético player becomes a symbolic representation of Real and the female Atlético fan is made the cup Real is about to hold in their hands, the prize they are about to win. It is Atlético that becomes the bystander on this second level established by the collage. However, perhaps because of the simplicity of the way in which the collage is created, it is open to different readings that depend on the mapping between the two levels, i.e., on the individual recipient's inclusion or exclusion of particular elements of the original picture as meaningful constituents of the collage. Thus, another reading of the three-party interaction is a

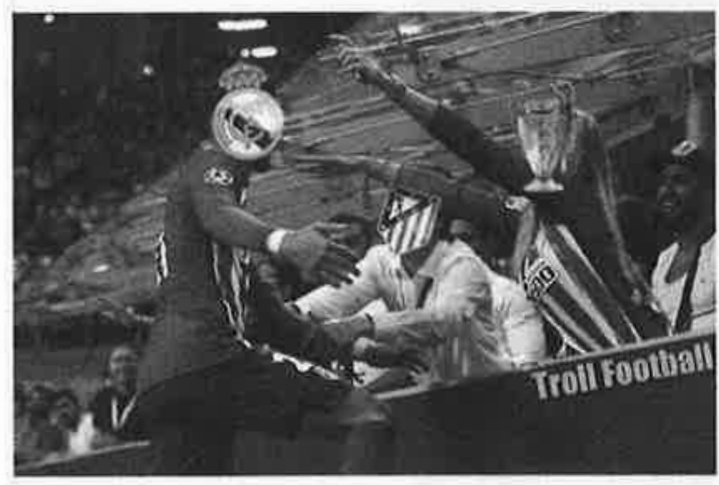

myltiz footballjokes

footballokes That says It all

Figure 11.6 Incongruous collage. Footballjokes, 29 May 2016. Instagram. 
reinterpretation of the male fan's grab of the player's arm as an attempt to stop Real from reaching the cup; and the collage of the female fan as Champions League cup can both be seen as an attribution of volition to the prize, which is thus consciously partial in the Real/Atlético rivalry, and/ or as a sexist mapping of prize to young woman on which the plausibility of the metaphorical links between the two levels depends. ${ }^{4}$

The collage shows how a simple yet effective manipulation of the picture enables the OP to make a humorous comment about the fact that contrary to the joyous scene of player and fan in the original picture, Atlético lost in the end, whereas Real won the competition. It is interesting to note here that while this humor strategy is clearly visual in nature, the humorous incongruity does not in fact reside either in the original picture or in the layer that the OP superimposed on it: What is incongruous here is the relationship between the two levels, which create a co-existence of two opposites.

\section{Strategy 5: juxtaposition of incongruous pictures}

For the final pictorial strategy we want to discuss here, the focus is also on the established relationship between the pictures it juxtaposes. An incongruity is created by combining two pictures that are in contrast with each other. This is the case in Figure 11.7, where-in the context of the young

\section{The reactions to Rashford's goal}

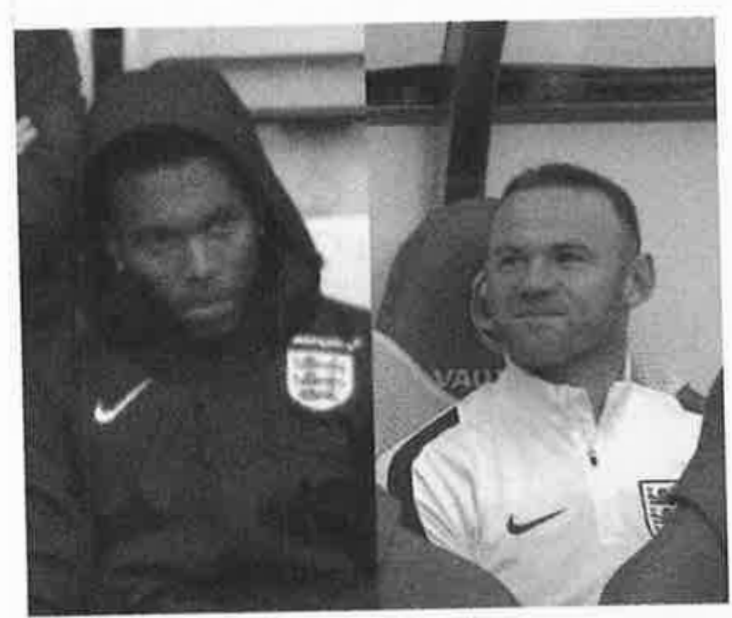

Following

porksoffootball The difference

Figure 11.7 Juxtaposed incongruous pictures. Perksoffootball, 3I May 2016. instagram. 
player Marcus Rashford scoring for the national team of England-two very different reactions by Rashford's team mates are contrasted: A smiling Wayne Rooney on the right faces a less than pleased Daniel Sturridge on the left. As was the case in Strategy 4, humor depends on the work done by the OP, that is, connecting the two pictures by putting them next to each other. Apart from the thus achieved incongruity, humor here can also be related to superiority, which is already present in the post. Essentially, the followers of the OP can laugh with them and the smiling Wayne Rooney about Sturridge who was at that time competing with Rashford for a place in the England squad at the Euro 2016. Thus, the successful communication of humor in this case requires for recipients to be familiar with the particular situation in which each of the depicted players found themselves at the time of posting. This allows recipients to make inferences about which players are in direct competition with each other and would therefore be happy or unhappy to see their teammate succeed.

\section{Text-related incongruities}

In the first five strategies, the OP constructed humor mainly in pictures or by establishing new relationships between pictures. Contrary to this, humor in the following two cases depends mostly on the work done in the captions or post texts.

\section{Strategy 6: humorous captions and post texts}

Despite the fact that visual materials are central to all four accounts, one strategy for OPs is to construct humorous incongruities linguistically and use imagery for purely illustrative purposes. In Figure 11.8, for instance, the OP pokes fun at Manchester City for spending too much money on an individual player and contrasts this with another successful club, Juventus, who have managed to sign a number of very successful players for free. Instead of listing the names of these players, the OP simply posts a picture of all of them.

\section{Strategy 7: humorous recontextualizations}

Another way of creating incongruity within captions or post texts is to present one or several non-humorous pictures and recontextualize them with the text. The post in Figure 11.9, for instance, unites two pictures of Real Madrid player Gareth Bale, one of his hair that appears to show a bald spot, and one where he celebrates the Champions League victory by putting the cup on top of his head. The caption recontextualizes this second picture and jokes that Bale uses the cup to cover up the bald spot shown in the other picture. 
Juventus got all these players for free.

Man City paid £49m for Raheem Sterling.

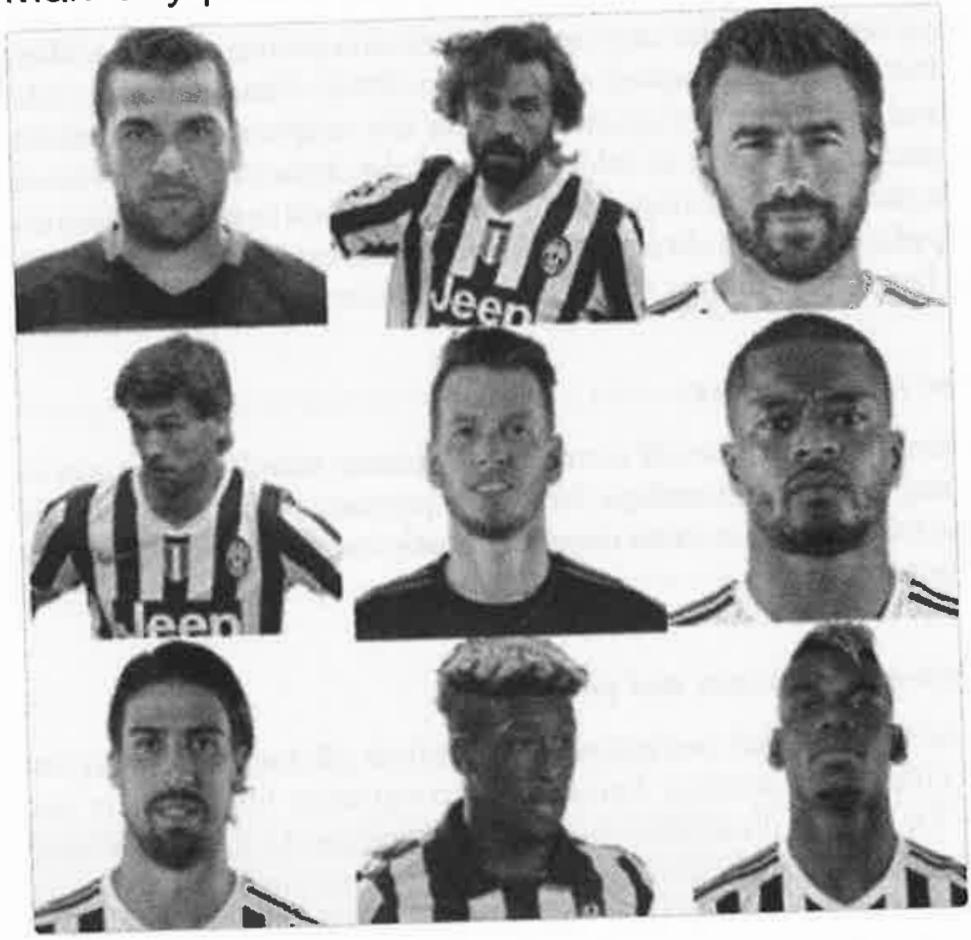

Figure 11.8 Humorous caption. Footyhumour, 5 June 2016. Twitter.

At first glance, this humor strategy appears to be very similar to Strategy 2, which uses captions to recontextualize non-soccer-related incongruities in pictures. In this case, however, there is no incongruity in the pictures per se, and the humorous effect on the followers depends on the caption providing an unlikely yet plausible interpretation of what the two images represent. While the two pictures are of course still essential to the post, the humorous incongruity is clearly established linguistically, and the caption can thus be identified as the primary mode in this strategy. 


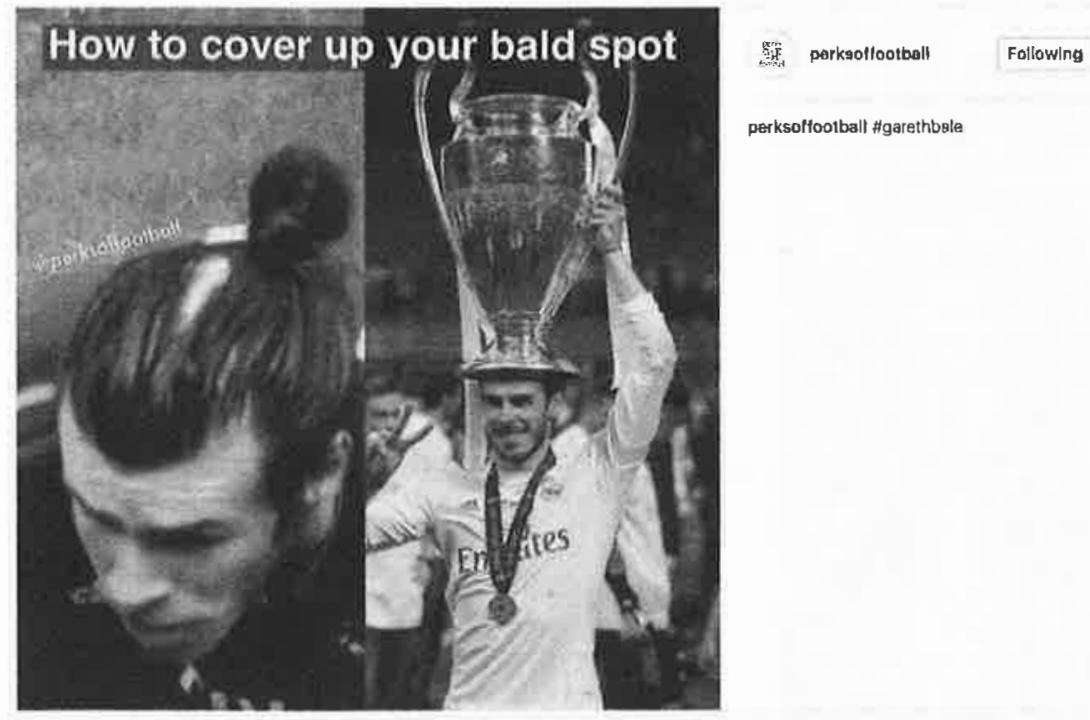

Figure 11.9 Humorous recontextualization. Perksoffootball, 31 May 2016. Instagram.

\section{Multimodal construction of incongruities}

In all the humor construction strategies we discussed so far, either the visual or the textual mode could be identified as the primary locus for the creation of humorous incongruities. In contrast, the final two patterns entirely depend on the interaction of the two modes.

\section{Strategy 8: juxtaposition of pictures and captions}

The first of these two types of truly multimodal humor-construction consists of non-humorous pictures and non-humorous captions. Humor emerges, however, when these are combined by the OP. Figure 11.10 illustrates this and shows three pictures that relate to personnel changes at Manchester United on the left as well as icons and text on the right. The pictures establish a chronology of former coach van Gaal leaving the club, Mourinho arriving as his replacement, and the transfer of Ibrahimović, which was rumored at the point in time of the post. The first two icons on the right seem to be made up of the logo of Nike, but they are used as check marks that identify the illustrated personnel changes as completed. The labels below the two marks specify the respective changes. The third icon, next to Ibrahimović, instead uses an icon that typically signifies within the domain of computers that a program is loading, which is indeed 


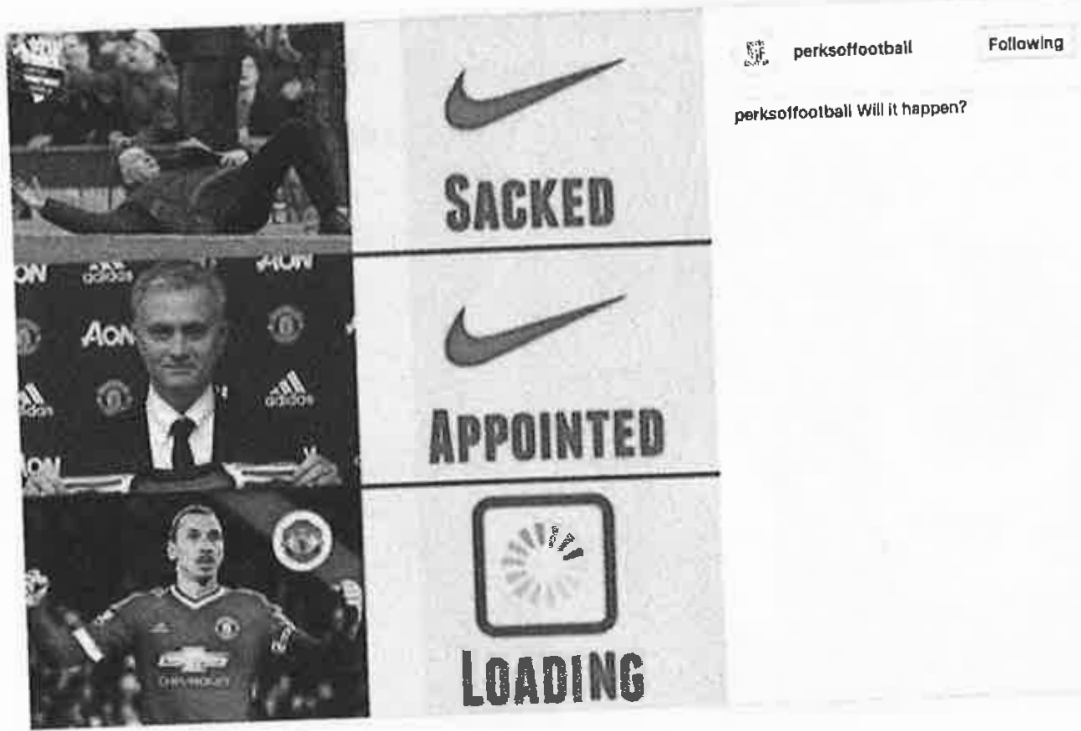

Figure 11.10 juxtaposed pictures and captions. Perksoffootball, I June 2016. Instagram.

confirmed by the label below it. In this case the incongruity is created between the frame of soccer and that of computer programs: Establishing the surprising connection ultimately amounts to a humorous way of representing the wait for the confirmation of the new signing.

Strategy 9: establishing and breaking a pattern

The final strategy that occurred in our data is reminiscent of a prototypical way in which jokes are structured. As Sacks (1974) already established, the pattern of three units-two similar ones and a different one-is one classic way in which jokes construct incongruities (see also Norrick, 1993). Similarly, types of posts on Twitter and Instagram create a sequence of pictures and captions, with two or more establishing a pattern, and the final one running against the expectations that this pattern evokes. In Figure 11.11, the first two pictures and captions establish that the two Champions League finalists, Real (left) and Atlético (right) are ready for the game, and the final, larger picture of the squad of Barcelona establishes in a colloquial register that they, too, are ready for the game. Since all three pictures show soccer teams and captions that state the teams are ready, it may seem at first glance that there is no unexpected element here and thus no incongruity. However, the different, 

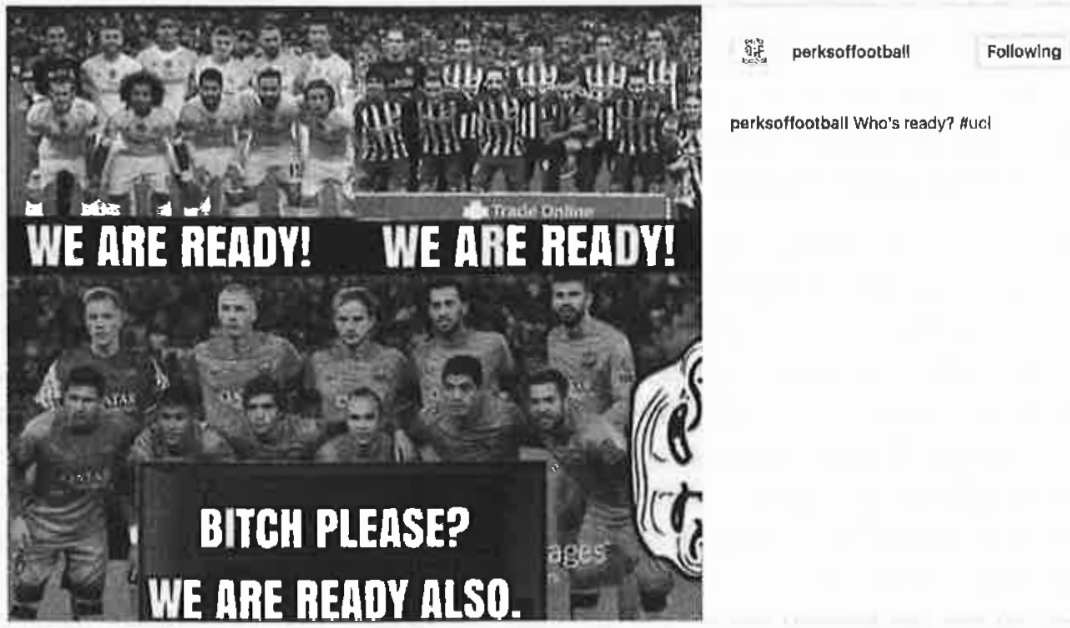

perksoffootball Who's ready? Hucl

Figure $1 / . \mid /$ Breaking the pattern. Perksoffootball, 3I May 2016. Instagram.

more colloquial phrasing is a first hint at a clear contrast between Barcelona and the two other teams: As Barcelona was already eliminated from the Champions League competition at that point, they quite simply had nothing to be ready for. Accordingly, the post serves to create humor at the expense of Barcelona and their fans.

\section{Summary of Analysis 1: humor construction in original posts}

The qualitative analysis of 80 posts from Twitter and Instagram accounts that focus on soccer-related humor revealed a range of different strategies that are used by OPs to construct humorous incongruities relevant to soccer-fans in their posts. While some of these strategies rely mostly on the finding of imagery that is humorous a priori, in which case the work of the OP mainly consists in finding and distributing the humorous material, in many cases humor only emerges due to the work done by the OP. On the one hand, this may include recontextualization in the sense that humorous materials from different domains are adapted to the soccer frame with the help of captions, or by establishing a connection between different frames by juxtaposing seemingly unrelated imagery. On the other hand, nonhumorous soccer-related images may be combined in creative ways to create humorous effects. The analysis furthermore showed that these posts are truly multimodal texts that depend both on linguistic and on visual 
modes, and allow humor construction in either of them or indeed between them, that is, humor that emerges only when text and image are successfully combined in order to create incongruities.

\section{Analysis 2: User responses to humorous soccer-related posts}

For the second part of the analysis, we examined a sample of responses to the Instagram and Twitter posts we have just illustrated in examples. Based on the number of comments, we limited this second analysis to the comments made to just one of the humorous soccer accounts on Twitter and Instagram each. As a result, the following findings are based on $643 \mathrm{com}-$ ments to the Twitter account FootyHumour and 1292 comments to perksoffootball on Instagram. Whereas the original posts in the first analysis could be treated as similar across the two platforms, there are some clear differences between the two platforms' presentations of comments that need to be addressed here. In the case of Instagram, comments appear listed under or to the side of the original post and are thus presented as part of that post. On Twitter, however, comments appear in the form of new tweets, which is to say that they-while being linked to the original post-exist independently and require Twitter users to move from comment to comment, in order to read them. ${ }^{5}$ While the first four sections of our summary and exemplification of comment patterns will discuss comments that occur on both platforms, the fifth and sixth will discuss phenomena that are exclusive to Twitter comments.

\section{Comments that do not directly relate to humor}

When it comes to the comments that are not directly related to the humorous incongruities in the original post, we find that followers first of all react to the $\mathrm{OP}$ and other users. In those cases where the OP asks a question in the initial post, this is taken up as a first pair part by many of the commenters, and-as is to be expected-they accordingly comply with a direct answer to the question as a second pair part in their responses.

In those posts where contrasting images are juxtaposed, we find comments that offer an opinion as to which of the two images, perceived as two choices offered by the OP, the commenter prefers. More generally, since most posts contain references to specific soccer players or teams, many comments offer evaluations of these figures and clubs of the sport. These evaluations in turn provide grounds for reactions by followers to other followers, which range from simple agreement or disagreement with the offered opinions to more ad hominem comments that either praise or insult other followers. 
As has become clear in the presentation of examples in "Analysis 1," most of the original posts require a significant amount of previous knowledge about soccer in general and recent important events in the soccer world more specifically. Accordingly, commenters do not always understand all references, which results in clarification questions in the comments. Similarly, some of the assertions made by OPs and followers alike lead to disagreements about their factual accuracy, which results in followers correcting one another in the comments.

There are also comments that can be described as purely interactional. These include early commenters simply claiming one of the first few spors in the sequential order of comments, which is done for instance by simply posting "first" or " 1 " as a first comment. Finally, there are many responses that tag other users, which is done by including an @ symbol followed by the respective user name. These comments serve to make the tagged users aware of the post and potentially trigger further responses by them.

\section{Humor appreciation}

When it comes to specifically humor-related comments, a first type of comment signals humor appreciation in some fashion. Often, this occurs in the form of typographic emoticons or image-based emojis, since Twitter and Instagram (as well as most major social networks) offer a wide range of such more elaborate emojis to their users. Based on our analysis, the cryinglaughing emoji (Figure 11.12) seems to be particularly popular. Appreciation of humor can also be encoded in language. This is done by transcribing laughter as any variation of "hahaha," by using an acronym such as LOL (laughing out loud), or by explicating humor appreciation, e.g., by asserting "that's funny." Typically, appreciation seems to be only encoded on one of the presented levels, i.e., either by using emoticons/emojis or by any of the linguistic strategies, but not by a combination of both.

\section{Humor construction in comments}

Rather than simply reacting appreciatively to humor, comments are also used to construct humorous incongruities. These incongruities may simply be call backs, i.e., repetitions of the humor created by the OP, but they also construct new incongruities, which typically relate to the same teams and/or players to which the OP made reference. In either case, the

Figure 11.12 Crying-laughing emoji. U+|F602: Face of tears of joy. Unicode. 
constructed humor often makes the presented players and teams the butt of the joke. In reaction to the post presented in Figure 11.10, for instance, one follower offers the following comment:

1 Ibrahimovic don't play on thursdays

First, this comment explicitly mentions Ibrahimović, who is presented in the original post, but the comment cleverly creates humor that targets not only him, but also the club he is at that point rumored to join, Manchester United. On the one hand, refusing to play on a certain day of the week ties in with the arrogance often associated with this player; on the other hand, Thursday is picked by the commenter because that is the day when Europa League games take place. Thus, the commenter also creates a humorous $\mathrm{jab}$ at Manchester United, who have not been successful enough to qualify for the superior Champions League, which would mean playing on Tuesdays or Wednesdays.

Finally, there are also some rare cases of simple language-based humor, for instance when a commenter makes fun of the name of Leicester City player Danny Drinkwater:

2 No drinkwater yes drinkchampagne

\section{Humor comment or question}

An interesting avenue of humor research is the study of failed and incomprehensible humor. With regard to incomprehensible humor, Bell (2013, p. 187) finds that for the most part, respondents "admit their lack of understanding openly." In the case of Twitter and Instagram comments, however, we would expect there to be much less pressure on followers to acknowledge their lack of comprehension, and we speculate that the default answer to failed and to incomprehensible humor will simply be the absence of a comment. Having said that, there are some responses in our data that explicitly confirm lack of comprehension:

\section{Don't get it}

4 What does it mean?

While there are only few occurrences of such admissions in our data, response to incomprehensible humor on social media would be an interesting topic for future research. 


\section{Stranded conversations}

Specifically on Twitter, one common type of response found in comments is stranded conversations in which commenters break into smaller conversations on their own, quite similar to the notion of schisming in face-toface multi-party interactions (Egbert, 1997; Sacks et al., 1974). The comments in Figure 11.13 are in reaction to an original post that reads, "I said Bale not Bailly you useless idiot..." featuring the photos of José Mourinho and one of Manchester United's scouts on the phone. The first user responds with a laugh token "haha" and commenting that "either way, @ericbailly 24 wouldn't be a bad signing," while tagging the OP as well as another user and one of the soccer players discussed. The two users then commence a three-turn conversation on their own regarding the details of the signing.

Although the two users break into a smaller, dyadic conversation, note that the tagged parties are notified of being tagged and have the choice of participating in this particular conversation. Similarly, other recipients of the original post can also view this stranded conversation and have the option of liking and retweeting particular turns, or even joining in. The specific strategies of schisming on social media platforms with such technological affordances, such as Twitter and Facebook, are also an interesting phenomenon that warrants further research.

\section{Multimodal comments}

Another unique phenomenon found on Twitter is the use of multimodal comments such as pictures and memes. Figure 11.14 below is an example
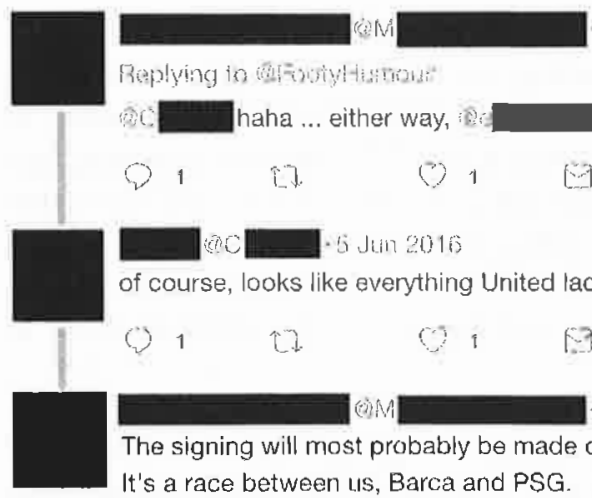

G. Jui 2016

Replying to afortytambut

haha ... either way, wouldn't be a bad signing.
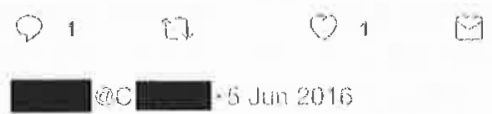

of course, looks like everything United lack at the back. Hopefully it's done ASAP
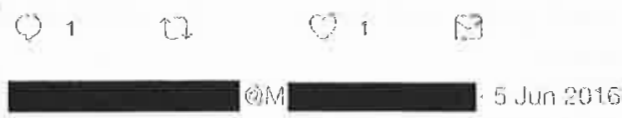

The signing will most probably be made official by the end of this coming weok. It's a race between LIS, Barca and $P S G$.

\%

Figure 11.13 Stranded conversation in comments. MoketeMoloantoa and Cobbz6, 5 June 2016. Twitter. 

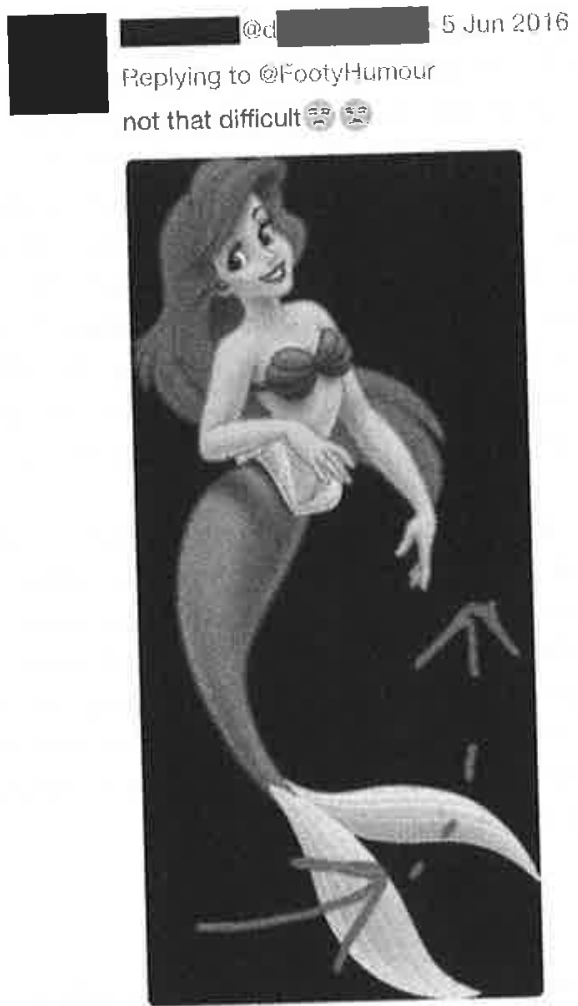

Figure 11.14 Multimodal comment. Danielellgam, 5 June 2016. Twitter.

of a multimodal comment responding to an original post that says, "Ronaldinho could nutmeg a mermaid \#SoccerAid2016." Responding to the OP, the recipient comments "not that difficult" with two frowning face emojis, accompanied by a picture showing a mermaid with a red line crossing through her tail. This seems to indicate that nutmegging a mermaid is not as challenging as the OP suggests. This comment is potentially critiquing the content of the original post and the use of the picture serves to illustrate the commenter's point.

Another example is the comment in Figure 11.15, which features a single meme with no verbal comment except tagging the OP. The original tweet, illustrated earlier in Figure 11.8, states that "Juventus got all these players for free. Man City paid $£ 49$ m for Raheem Sterling," while posting the photos of nine soccer players. The comment itself is a meme featuring Spiderman sick in bed with the caption "THIS POST GAVE ME CANCER, EBOLA AND AIDS," which receives 12 likes. The meme serves 

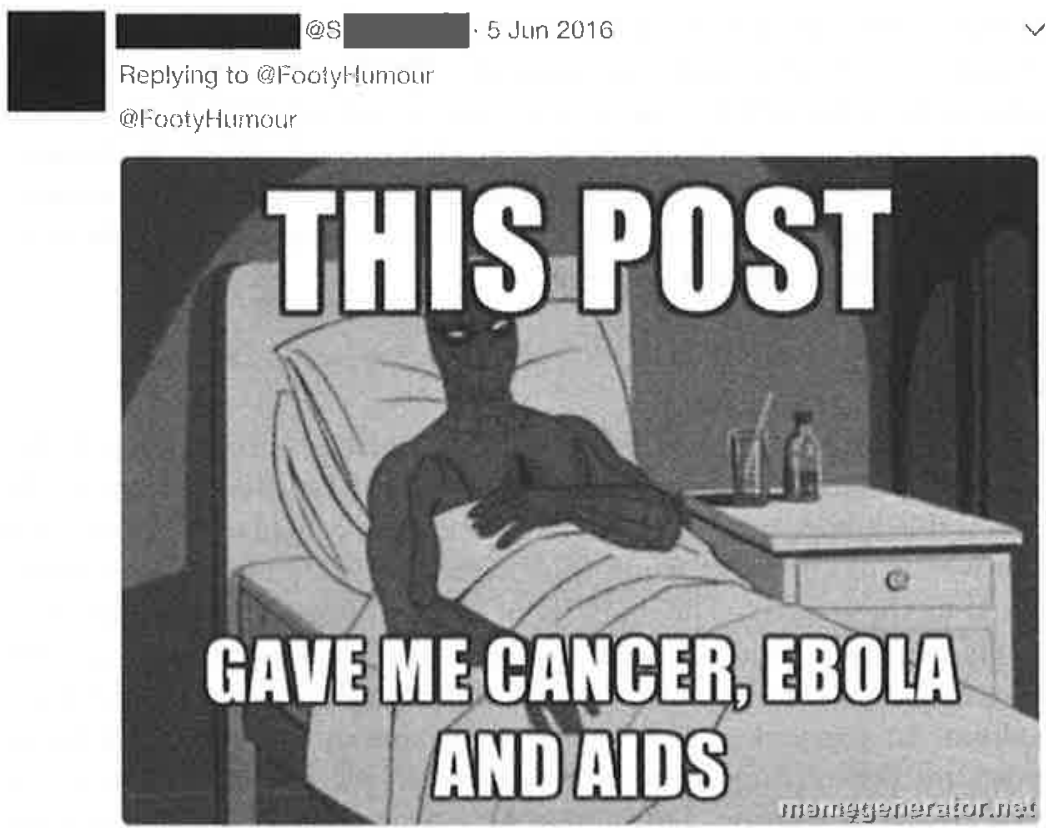

Figure /1.15 Multimodal comment (Twitter: Footyhumour). Sambob I80, 5 June 2016. Twitter.

to illustrate the strong impact of the original post (i.e., "it kills me"), be it of its humorous effect, or its poignancy, or perhaps the lack of humor.

Although not many instances of multimodal comments are present in our data, they enable the audience to comment in ways more complex than a brief verbal comment, which allows more equal participation and encourages creative use of the comment space.

\section{Summary of Analysis 2: user responses}

The second part of the analysis, which addressed user reactions to humorous posts, illustrates first of all that there are a range of response patterns that are not directly linked to humor. Besides offering evaluative comments related to specific players or teams, more importantly for the central questions of this chapter, commenters were shown to signal humor appreciation in different ways and even to construct new humorous incongruities in their comments. In cases where they inferred the intention by the OP to be humorous but did not understand the post's humor, commenters also explicated their lack of comprehension and asked the OP for clarification. We have also observed that there are humor response patterns that are exclusive to Twitter. Twitter commenters have the option to post 
multimodal comments, which means that they have the option to construct further instances of multimodal incongruities. Finally, the difference in presentation, with responses to tweets being more independent from the OP than those on Instagram, also leads to stranded conversations on Twitter. More generally, we find that Twitter facilitates more discussion and interaction among recipients and enables them to make creative contributions to humor as a type of support strategy.

\section{Conclusion}

We analyzed 80 posts on two Twitter and two Instagram accounts dedicated to soccer-related humor as well as just fewer than 2000 comments, in order to establish how posters used the multimodal affordances of the two social networks to construct humorous incongruities, and how commenters reacted to these posts. The analysis of original posts showed that even within this relatively small sample, a range of strategies are employed by posters, which make full use of the multimodal affordances provided by the medium. In particular, posters based the humorous incongruities in their posts on pre-existing humor they found in pictures and videos, but they also creatively constructed humor by juxtaposing visual and textual materials from the domain of soccer as well as from other domains. Often, humor is thus not or not only a product of funny pictures and funny texts, but also the result of a relationship between different stimuli that is only established due to the work done by the broadcasting party.

When it comes to the responses to posts, our data shows that commenters actively engage with OPs by signaling humor appreciation and recognition in different ways, but also by communicating explicitly that they did not understand a particular humorous instance. Furthermore, commenters also interact with each other-sometimes in direct relation to the topical humor of the OP and at other times more independently. On Twitter in particular, responses which appear in the form of new tweets that are linked to the original tweet can lead to separate dyadic conversations, which also indicates that Twitter as a social platform encourages more active participation in humor construction by followers than is the case on Instagram. However, both networks enable not only posters, but also their followership to play an active role in humor construction. Even an action as simple as tagging another user contributes to active participation and multiple authorship of soccer-related CMC humor, since it invites other users to join the interaction and contribute to the humorous event, be it by simply liking or retweeting the original post, by showing their appreciation, criticism or incomprehension, or even by contributing more humor in support of the OP. Thus, the participation role of followers is not limited to that of passive recipients: They are involved as agents who create humorous content by and for themselves, which means that the 
conversations that ensue can be described as polylogues (Marcoccia, 2004), in which followers encourage other users to co-construct further humorous instances. The resulting humorous polylogues underline that humor in this domain, although managed by broadcasters, is a collaborative effort that involves broadcasters, followers, and other users.

\section{Appendix}

Note: some URLs might be inaccessible due to accounts that have since been deleted.

\section{Notes}

1 While the number of participants is of course finite at any given moment in time and restricted to those that have the technological means to access the particular form of online communication in question, the claim of a potentially infinite audience includes the fact that interactions on social media are on record, i.e., that it is not foreseeable when or if they cease to be accessible to new audiences.

2 In this section, which focuses on original posts, the figures present the respective post as it appeared on our screen, with comments whited out.

3 Identifying a man kissing another man as incongruous of course in no way reflects the value systems of the authors of this chapter. However, responses to this post such as "Ha gayyy ..." and "The one that is not gay ..." reveal that some of the followers do indeed evaluate the pictures as incongruous because of their representation of male soccer players kissing male colleagues or fans.

4 We would like to thank the editors for pointing out the alternative readings of this collage. They not only show additional potential for this particular post, but also highlight yet again that humor is ultimately constructed by individual recipients. While most of the posts we examine here are fairly unambiguous at least when it comes to the main incongruity that is being constructed, others allow a range of different interpretations for recipients and only encode some cues that allow researchers to infer the OP's intent.

5 There is of course a range of devices and, in the case of Twitter also a range of software, through which posts and comments can be read. The individual choice of soft- and hardware will influence the reading experience and may in some cases relativize the distinctions we made here. However, the differences hold true at least when users access posts through the official Twitter and Instagram apps on their smartphone or computer.

\section{References}

Attardo, S. 1994. Linguistic theories of humor. Berlin: Mouton de Gruyter.

Attardo, S., Pickering, L. and Baker, A. 2011. Prosodic and multimodal markers of humor in conversation. Pragmatics \& Cognition. 19(2), pp. 224-247.

Baym, N.K. 1995. The performance of humor in computer-mediated communication. Journal of Computer-Mediated Communication. [Online]. 1(2), [no pagination]. [Accessed October 12, 2017]. Available from: https://pdfs.semantic scholar.org/e277/1956aadd617afc48a548c2da50b438c03313.pdf. 
Bell, N.D. 2009. Responses to failed humor. Journal of Pragmatics. 41(9), pp. 1825-1836.

Bell, N.D. 2013. Responses to incomprehensible humor. Journal of Pragmatics. 57, pp. $176-189$.

Bolander, B. and Locher, M.A. 2014. Doing sociolinguistic research on computermediated data: A review of four methodological issues. Discourse, Context and Media. 3(1), pp. 14-26.

Butterworth, M.L. 2014. Social media, sport, and democratic discourse. In: Billing, A.C., Hardin, M. and Brown, N.A. eds. Routledge handbook of sport and new media. New York: Routledge, pp. 32-42.

Chovanec, J. and Dynel, M. 2015. Researching interactional forms and participant structures in public and social media. In: Dynel, M. and Chovanec, J. eds. Participation in public and social media interactions. Amsterdam: John Benjamins, pp. $1-23$.

Cleland, J. 2014. Racism, football fans, and online message boards: How social media has added a new dimension to racist discourse in English football. Journal of Sport and Social Issues. 38(5), pp. 415-431.

Coates, J. 2007. Talk in a play frame: More on laughter and intimacy. Journal of Pragmatics. 39(1), pp. 29-49.

Draucker, F. 2015. Participation structures in Twitter interaction. In: Dynel, M. and Chovanec, J. eds. Participation in public and social media interactions. Amsterdam: John Benjamins, pp. 49-66.

Egbert, M.M. 1997. Schisming: The collaborative transformation from a single conversation to multiple conversations. Research on Language and Social Interaction. 30(1), pp. 1-51.

Fillmore, C.J. and Baker, C. 2009. A frames approach to semantic analysis. In: Heine, B. and Narrog, H. eds. The Oxford handbook of linguistic analysis. Oxford: Oxford University Press, pp. 313-340.

Glenn, P. 2003. Laughter in interaction. Cambridge, UK: Cambridge University Press.

Hay, J. 2001. The pragmatics of humor support. Humor. 14(1), pp. 55-82.

Jefferson, G. 1979. A technique for inviting laughter and its subsequent acceptance/ declination. In: Psathas, G. ed. Everyday language: Studies in ethnomethodology. New York: Irvington, pp. 79-96.

Lee, F.L. 2005. Spectacle and fandom: Media discourse in two soccer events in Hong Kong. Sociology of Sport Journal. 22(2), pp. 194-213.

Marcoccia, M. 2004. On-line polylogues: Conversation structure and participation framework in internet newsgroups. Journal of Pragmatics. 36(1), pp. 115-145.

Milner, R.M. 2013. Pop polyvocality: Internet memes, public participation, and the Occupy Wall Street movement. International Journal of Communication. 7, pp. 2357-2390.

Norrick, N.R. 1993. Repetition in canned jokes and spontaneous conversational joking. Humor. 6(4), pp. 385-402.

Norrick, N.R. 2003. Issues in conversational joking. Journal of Pragmatics, 35(9), pp. 1333-1359.

Sacks, H. 1974. An analysis of the course of a joke's telling in conversation. In: Bauman, R. and Sherzer, J. eds. Explorations in the ethnography of speaking. Cambridge, UK: Cambridge University Press, pp. 337-353. 
Sacks, H., Schegloff, E.A. and Jefferson, G. 1974. A simplest systematics for the organization of turn-taking for conversation. Language. 50(4), pp. 696-735.

Suls, J.M. 1972. A two-stage model for the appreciation of jokes and cartoons: An information-processing analysis. In: Goldstein, J.H. and McGhee, P.E. eds. The psychology of humor: Theoretical perspectives and empirical issues. New York: Academic Press, pp. 81-100.

Tannen, D. 2007. Talking voices: Repetition, dialogue, and imagery in conversational discourse. 2nd ed. Cambridge, UK: Cambridge University Press. 
Routledge Research in Sport. Culture and Society

\section{THE AESTHETICS, POETICS, AND RHETORIC OF SOCCER}

Edited by

Ridvan Askin, Catherine Diederich, and Aline Bieri

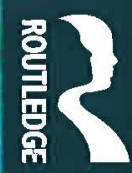




\section{The Aesthetics, Poetics, and Rhetoric of Soccer}

Edited by Ridvan Askin, Catherine Diederich, and Aline Bieri 
First published 2018

by Routledge

2 Park Square, Milton Park, Abingdon, Oxon OXI4 4RN

and by Routledge

71I Third Avenue, New York, NY 10017

Routledge is an imprint of the Taylor \& Francis Group, an informa business

(C) 2018 selection and editorial matter, Ridvan Askin, Catherine Diederich, and Aline Bieri; individual chapters, the contributors

The right of Ridvan Askin, Catherine Diederich, and Aline Bieri to be identified as the authors of the editorial matter, and of the authors for their individual chapters, has been asserted in accordance with sections 77 and 78 of the Copyright, Designs and Patents Act 1988.

All rights reserved. No part of this book may be reprinted or reproduced or utilised in any form or by any electronic,

mechanical, or other means, now known or hereafter invented, including photocopying and recording, or in any information storage or retrieval system, without permission in writing from the publishers.

Trademark notice: Product or corporate names may be trademarks or registered trademarks, and are used only for identification and explanation without intent to infringe.

British Library Cataloguing-in-Publication Dato

A catalogue record for this book is available from the British

Library

Library of Congress Cataloging-in-Publication Data

A catalog record has been requested for this book

ISBN: 978-0-8I 53-8573-8 (hbk)

ISBN: 978-I-35I-|8040-5 (ebk)

Typeset in Sabon

by Wearset Ltd, Boldon, Tyne and Wear 